\title{
Automatic extraction of rivers from satellite images using image processing techniques
}

\author{
Carey Ciaburri $^{1 *}$, Monica Kiehnle-Benitez ${ }^{1}$, Alaa Sheta ${ }^{2}$ and Malik Braik ${ }^{3}$ \\ Department of Physics, Southern Connecticut State University, New Haven, USA ${ }^{1}$ \\ Department of computer science, Southern Connecticut State University, New Haven, USA ${ }^{2}$ \\ Department of computer science, Al-Balqa Applied University, Salt, Jordan ${ }^{3}$
}

Received: 13-April-2020; Revised: 20-May-2020; Accepted: 22-May-2020

(C)2020 Carey Ciaburri et al. This is an open access article distributed under the Creative Commons Attribution (CC BY) License, which permits unrestricted use, distribution, and reproduction in any medium, provided the original work is properly cited.

\begin{abstract}
Automatic extraction of water bodies from satellite imagery has been broadly studied for many reasons, including mapping of natural resources (i.e., forest and water resources), drinking water supplies, food production, agricultural planning, and disaster management. With the growth of global warming, it became essential to maintain the sustainable management of these resources for the preservation of human life. Several methods attempted to allocate water bodies from different satellite imagery in both spatial and spectral domains. In this paper, we present an automatic segmentation method to extract the water body from Landsat satellite imagery. The proposed segmentation approach consists of several stages, including histogram stretching, de-correlation, binarization of the image, and clutter removal using morphological operations. The segmentation results are promising.
\end{abstract}

\section{Keywords}

Rivers detection, Satellite images, Enhancement, Segmentation, Recognition, De-correlation.

\section{Introduction}

River water is an essential natural resource that is a vital component affecting the global carbon cycle and climate change [1]. Thus, river water is an essential component of the environment, which has a vital role in the survival of humans, plants, animals, as well as economic development. Generally, river water is utilized for multiple purposes such as agriculture, drinking, hydropower projects, etc. Accordingly, it is important to take care of the rivers and identify the areas of water pollution in the river as well. However, extracting rivers from satellite imagery remains a difficult problem because rivers have complex shapes with diverse branches and directions [2]. Synthetic Aperture Radar (SAR) images have been used growingly for water monitoring, due to their ability to register images independently of weather conditions [3]. Many algorithms for extracting water using SAR images rely on optimum thresholding techniques [4-6].

*Author for correspondence

32
However, these simple techniques generated many false classification results, particularly when the bodies of water are small. For example, five different thresholding methods applied to three satellite images to determine the most effective technique for image segmentation was presented in [7]. These threshold methods include the P-tile method, Histogram Dependent Technique (HDT), the Mean method, and the Edge Maximization Technique (EMT). Al-Amri and Kalyankar [7] found that HDT and EMT methods yielded the best results. HDT technique is histogram-based, where the histogram depends on the value of the threshold. The allocated threshold value separates the area of the image where the feature of interest blends into the background. EMT method is used when there is a distinct contrast in illumination between the feature of interest and the background. Gong et al. [8] proposed an automatic adaptive threshold segmentation approach based on the fuzzy c-means algorithm and the Otsu threshold method to extract tidal creeks.

Sakurai-Amano et al. [9] presented a fully automated method to extract the location of narrow rivers in tropical rain forests-based images collected from JERS-1 SAR images. Their method was examined by 
comparing the outcomes from optical JERS-1 VNIR in the Amazon basin and the Congo basin. Commonly, images obtained via remote sensing suffer from poor contrast and low resolution [10]. The importance of overcoming these challenges and successfully processing satellite images for research is relevant to fields such as astronomy, agriculture, and environmental industries and water management [11].

As discussed above, water management research has particularly gained momentum as environmental bid, including growing overpopulation and pollution surrounding water resources are on the rise. Protecting natural water supplies has become a top priority as water is an important life-sustaining resource [12]. In such a context, the detection of water bodies and extracting them from satellite imagery is beneficial operations for many designing and evolution activities such as coastline mapping, river erosion mapping in addition to water resource management. Scientific research in the field of water resources management and planning worldwide has explored various satellite data to understand the spatial, spectral, and temporal characteristics to produce maps of land with a special interest in water bodies [13]. Detecting water resources was not only limited to the use of satellite remote sensing images, but an Unmanned Aerial Vehicle (UAV) was utilized for the same purpose [14, 15]. For example, Randazzo et al. [16] proposed an image processing framework for coastal extraction as well as shallow water depth on the GeoEye-1 satellite image and mosaic image collected by a drone on the coast of San Vito Lo Capo. Several researchers have endeavored to analyze river water using machine learning tools and high-resolution satellite images. In this regard, we review some popular works of water body extraction during the period from 2015 to 2020 .

There are several published works of various methods involving the segmentation of features in satellite images. Although not much has been explored with extracting rivers, in particular, several objects have been successfully detected and extracted. Dhanachandra et al. [17] successfully extracted rivers from satellite images and the two methods were explored. The first included the extraction of rivers using a color histogram technique, and hill-climbing algorithm and k-means clustering method. The second one included the use of the thresholding of a grayscale image and morphological erosion. The results achieved were reasonable based on both methods. Syrris et al. [18] discussed the importance of image enhancement and contrast adjustment when extracting features from low-resolution satellite data. The authors built a case study that encompasses the use of enhancement techniques such as linear and decorrelation stretching. They showed that low-resolution satellite imagery could be improved or "corrected" and thus more effective when extracting features.

Jiang et al. [19] adopted a multilayer PerceptronArtificial Neural Network (ANN) to determine the surface water in Landsat 8 satellite imagery. A study presented in [20] used ANN and a threshold technique with a set of mathematical morphology operations to extract lakes from satellite imagery. A segmentation approach based on Convolutional Neural Networks (CNN) was used for extracting water bodies from remote sensing images with high resolution that were gathered from Google earth imagery [21]. A deep learning approach was developed in [22] to interactively extract water bodies, farmlands, forests, and other nonartificial regions from satellite images with high resolution. Their approach was performed on two datasets with different object types and varied, complicated scenes [22].

Meng et al. [23] presented an automated Lake and Reservoir Extraction Process (LREP). This method implemented a Modified Two-Mode (MTM) method for extracting water from partial images using 154 Gaofen-2 images covering Zigong, Xianning, and Liaoyuan cities. A study given in [24] reported an enhanced deep convolutional encoder-decoder network to extract body water from remote sensing images through the application of superpixel segmentation and conditional random fields.

Rishikeshan and Ramesh [25] presented a flexible Mathematical Morphological (MM) driven approach for extracting water bodies from much different satellite imagery of different spatial resolution. An operational automated water extraction method was used for the extraction of water bodies for a large number of lakes in China using Landsat 8 land images. This method does not demand the determination of histogram peaks, but instead searches for the minimum values in the threshold range to arrive at an automated dynamic threshold [4].

Moreover, a variety of image processing and machine learning methods have been adopted for the detection of pollution in river water. Analysis of different 
satellite sensors, image processing methods and many classification methods to classify river water was provided in [26]. Navarro et al. [27] presented an unsupervised approach using the local Moran index of spatial association combined with morphological processes for extracting inland water bodies. Dereli and Tercan [28] developed a method to detect shoreline changes of Lake Salda in Turkey from the year 1975 to the year 2019. A segmentation approach using the stepwise thresholding technique was drawn to track and map the change in the extent of surface water using SAR images in Ontario, Canada from 2008 to 2016 [5].

A method combining Google Earth Engine (GEE) and a multiscale CNN was presented in [29] for detecting water from Landsat images. An approach that combined global salience features, multi-feature fusion method based on principal component analysis, and an Active Contour Model (ACM) to extract rivers from SAR imagery was proposed in [30]. In [31], the authors presented an assessment to the accuracy of a new automated water detection method by integrating the automatic water extraction index, GEE platform, and Landsat 8 OLI data throughout the years 2014, 2015, 2016, 2017 and 2018 to study the case of New Zealand. The proposed image fusion method was successfully utilized to test the real changes of the lakes at a more accurate scale.

In light of the importance of automatic extraction of water bodies from satellite images, the main goal of this work is to present an automatic method to extract the water body from Landsat satellite images based on a method consisting of several image processing stages. These stages include enhancement, segmentation, and clutter removal of images. We used several images collected from the Connecticut River in the state of Connecticut. The images were acquired through Google Earth at an altitude between 14,000 and 15,000 meters. The paper is structured as follows. In Section II, we provide a discussion of our proposed methodology. Section III describes the experimental setup and the developed results. Finally, we provide our conclusions and future work.

\section{Proposed methodology}

Our proposed method for extracting the water body from a Landsat satellite imagery is based on several steps. They include enhancement, binarization, morphological operation, and clutter removal. Image enhancement and pre-processing techniques include histogram equalization and de-correlation stretching. Image enhancement is often thought of as only visually improving an image. However, by improving the contrast and overall quality of the raw image before the segmentation process, one will potentially yield better results [18]. Thresholding is also an essential step to image segmentation as it aids in the separation of objects in the foreground from the background. Converting an image to grayscale, then to binary by selecting a threshold value, is key to foreground object and feature detection [32].

Segmentation plays a critical role in providing the ability to analyze and gain information from an image. This process involves the separating and grouping of pixels based on similar characteristics allowing features to have higher contrast and to become distinguishable. The process and technique used to achieve segmentation largely depend on the task at hand [32]. Image segmentation can be performed on either grayscale or color images, making it useful in several image processing applications. A survey of global thresholding methods for mapping, open water bodies was presented in $[33,34]$. A recent study in the Tianjin Binhai New Area was implemented to extract the surface water using a Modified Normalized Difference Water Index (MNDWI) method to extract surface water and detect its change [35]. The finding claims that the environment evaluation method shows that the water quality of the Haihe River section was poor. It was suggested to strengthen the environmental protection protocol in this area.

\section{A.Enhancement via decorrelation}

Color enhancing is an essential process for object detection in satellite images. To show multispectral images that fall outside the range of the human spectrum, an appropriate transformation must be rendered for representing the data in color. Therefore, need to use an appropriate method to enhance the image for better detection of water resources locations such as rivers.

Decorrelation stretching is one method that can be used to enhance the color domains of an image with considerable band-to-band correlation. The inflated colors expand the visual understanding of the image and make feature perception clearer. In the past, the decorrelation stretching was explored by many researchers as a successful tool to handle multispectral imaging that can space channels in the spectral domain, and hence, achieve exceptionally high inter-channel correlation [36, 37]. In our proposed method we plan to use the decorrelation method. 
The decorrelation stretching process is used to enhance (i.e., stretch) the color differences in satellite images. The method depends on removing the interchannel correlation obtained in the image pixels; this is why we call the process "decorrelation stretch". The decorrelation stretch is a procedure that enhances the three color bands independently from each other. The RGB space represents only one of the possible bases for the color space. If we think of R, B, G, as $\mathrm{X}, \mathrm{Y}, \mathrm{Z}$ for a normal Cartesian space, then every point in the color space has an $R$ coordinate, a $G$ coordinates, and a B coordinate. Moving along $\mathrm{R}$ increases "redness" from a very dark red to a very light red, and similarly, for $\mathrm{G}$ and $\mathrm{B}$.

The decorrelation stretching algorithm normalizes each band and then rotates the bands into eigenspace. The algorithm finds the rotated orthogonal coordinate system along which the colors in the images are maximally extended and then stretches the colors along with those coordinates to the maximal extent. In this step, the bands lose their relationship (or correlation) to each other. Then, the bands are rotated back to the original RGB space, where they retain their normalized and decorrelated values [38]. The net impact of the process is to get an output image in which the pixels are well distributed between all possible colors while maintaining the relative meaning of hue, saturation and intensity components of the input image [36]. Figure 1 shows the block diagram of the proposed methodology. In Figure 2, we show a satellite image utilized by decorrelation stretching along with the histogram of both images. To show how the band of color stretched, we have shown the color scatter plot before and after stretching in Figure 3.

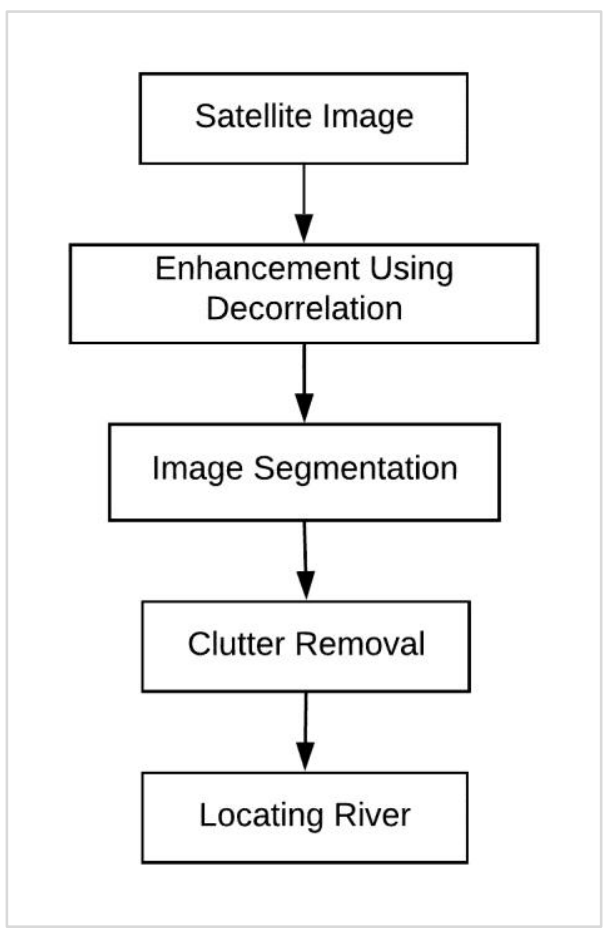

Figure 1 Block diagram of proposed methodology

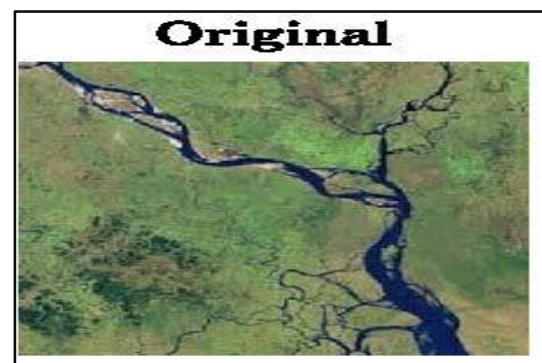

Decorrelation

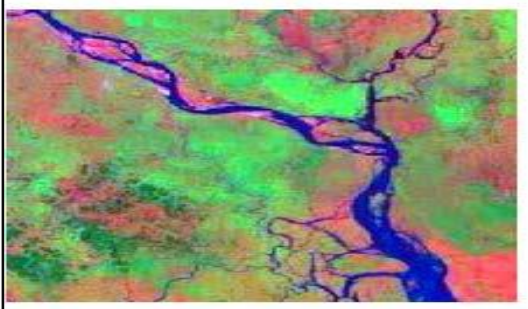

Original Historgram

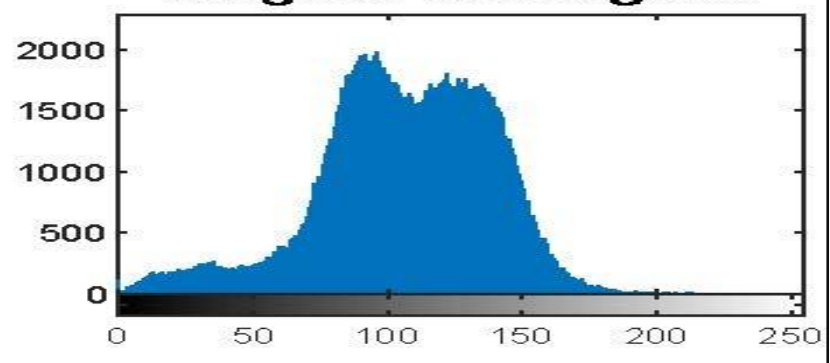

Decorrelation Histogram

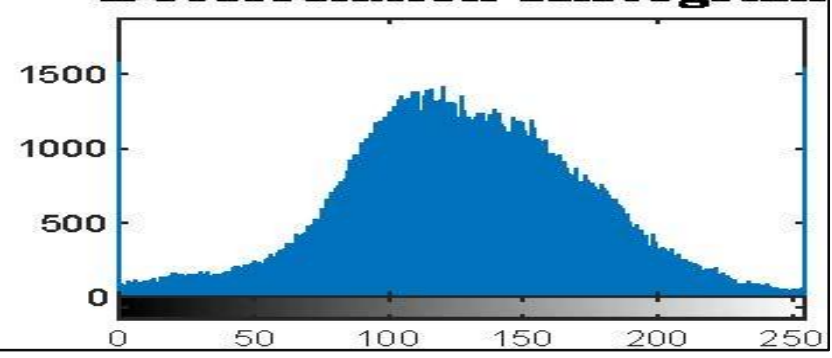

Figure 2 (a) Original satellite image (b) decorrelated image 

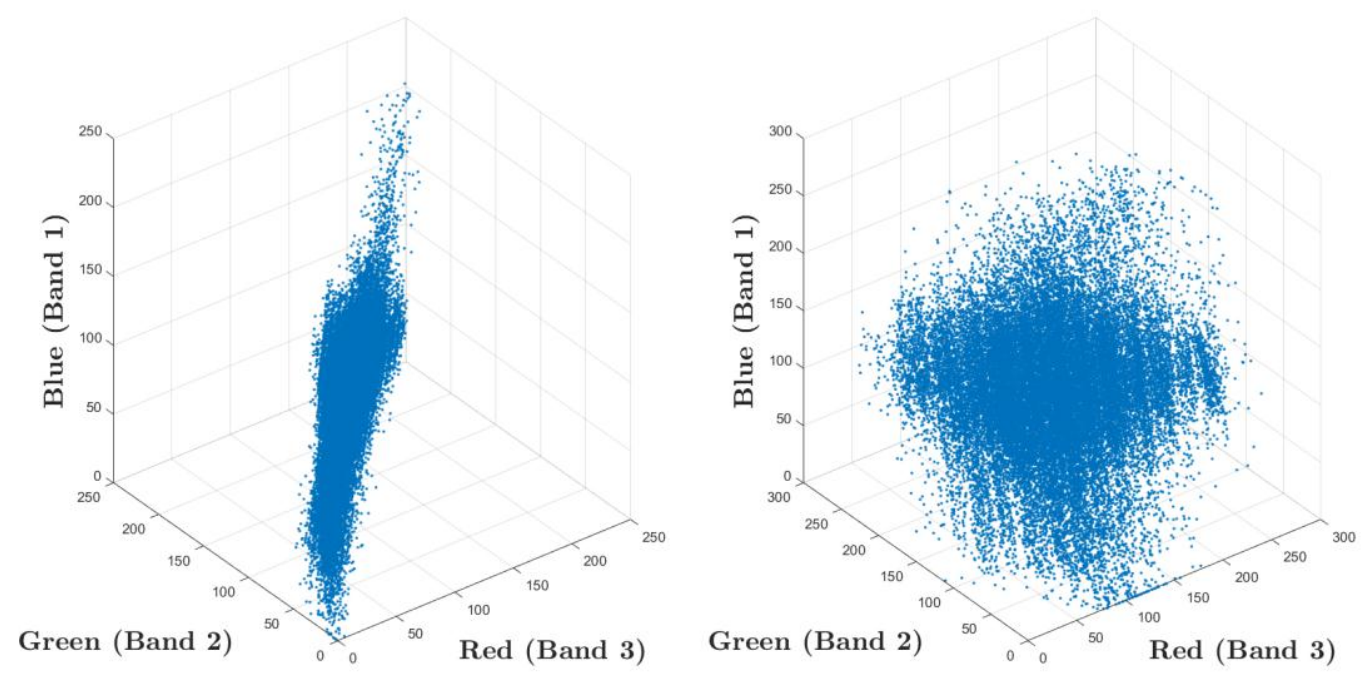

Figure 3 Color scatterplot before (a) and after (b) decorrelation

\section{B. Segmentation}

Image segmentation is the process of splitting an image of interest into several regions or categories. Each region is corresponding to different objects. Every pixel in an image will be classified based on a pre-defined criterion. In the case of grayscale images, the goal is to identify the similar grayscale pixels and recognize the relationship of these pixels as an object. Objects can later be recognized based on a set of selected features extracted from clustered objects.

Image segmentation plays a key role in many image processing tasks throughout partitioning an image into homogenous areas or objects that share similar properties. The extracted regions of interest of a particular image become more meaningful and can be utilized for further analysis and processing [39]. There exist well-known segmentation techniques reported in the literature such as:

- Threshold-based Segmentation

- Edge-based Segmentation

- Region-based Segmentation

- Clustering-based Segmentation

Threshold-based segmentation: The threshold-based segmentation method is modest and efficient in segmenting grayscale images that can fulfill the segmentation task by comparing the intensity of the image understudy to one or more intensity thresholds [40]. Threshold-based methods are categorized into global and local thresholding techniques [41].
- Global thresholding is the best option for dividing the objects and backgrounds if an image has objects with homogeneous intensity or the contrast between the objects and the background is too large. Choosing the proper threshold becomes hard when the contrast of an image is low.

- On the other hand, local thresholding can be identified by estimating a threshold value for different regions from the intensity histogram. The threshold values of local thresholding can be generally estimated using local statistical properties such as the mean intensity value [42].

An image described as $\mathrm{f}$ with grayscale levels from 0 to 255 consists of a light object and a dark background, with pixels distributed in two dominant modes, where $f(x, y)$ represents the pixel intensity at coordinates $(x, y)$. A single threshold value, $\mathrm{T}$, is used to group the pixels of $f$ into black (i.e., with a value of 0 ) and white (i.e, with a value of 255), which results in extracting the region of interest within the image for segmentation. An increase in the threshold values gives the increased number of intensity values in the segmented image. For the application of thresholding-based segmentation technique, it is necessary to apply the correct threshold values to achieve appropriate segmentation results. Specifically, the pixels below threshold $T$ will be categorized as class $C 1$ with the rest of pixels as class C2 as shown in Equation 1, where these two classes differ from each other. 
$g(x, y)=\left\{\begin{array}{l}C 1, \text { if } f(x, y)>T \\ C 2, \text { if } f(x, y) \leq T\end{array}\right.$

In many cases, the threshold value of $t$ is selected manually by the user. It is more likely a trial and error process to explore various objects in an image. The best value of $t$ can help to identify the objects of interest. Histogram Thresholding algorithm can be described briefly as shown in Algorithm 1.

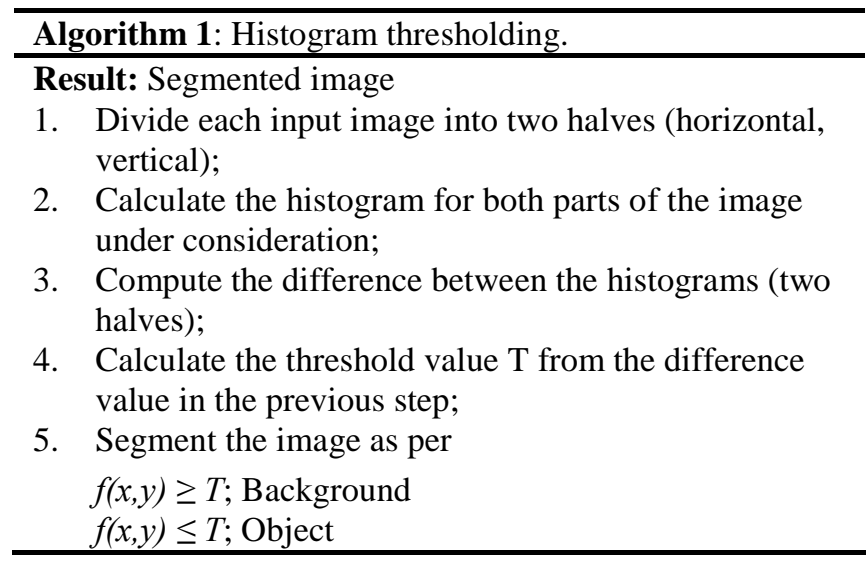

\section{Clutter removal}

Small unwanted objects in the image are removed by using an area opening morphological operator. Per the image characteristics, a maximum pixel amount to be imputed. As a result, all objects having fewer than the selected number of pixels will be removed from the image.

\section{Experimental results}

In this section, we present the results of our proposed methodology, which consists of image enhancement, segmentation, and clutter removal, with the final output, which is the segmented river.

\section{A. Data set}

The dataset used in this research consisted of images collected from the Connecticut River in the state of Connecticut. The images were acquired by Google Earth at an altitude between 14,000 and 15,000 meters. The results of the proposed segmentation method for a selected image are shown in Figure 4.

\section{B. Analysis of results}

Figure 4 (a) shows the original satellite image that is targeted to a segment based on the proposed image segmentation approach. In the first phase of the image segmentation problem is the application of the enhancement phase. In this phase, a decorrelation and stretch algorithm is applied to the original satellite image in Figure 4 (a). The output of this enhancement phase is a decorrelated RGB band with a contrast stretch as exhibited in Figure 4 (b). The next phase of the image segmentation problem is to segment the image regions. In this phase, first, the image is converted to binary, and then a global thresholding technique with a thresholding level, $\alpha$, of an arbitrary value between 0 and 1 is applied to the enhanced image. The result of the thresholding segmentation phase is presented in Figure 4 (c).

In the final phase of the image segmentation method, the clutter removal procedure is applied. In this phase, clutter with specific sizes $\beta$ is removed based on the characteristics of the image. In the case presented in Figure 4, $\beta$ took values up to 1000 pixels. Finally, the location and outlines of the detected River are shown in Figure 4 (d). The proposed image segmentation method was also applied to several different river images with various degrees of complexity and in various shapes. A set of examples of segmented satellite images is shown in Figures 5 to Figure 8.

The original images in Figures 5-8 show normal variation with a moderately complex background. Each image in Figures 5-8 (b) - (d) shows a separate phase of the proposed image segmentation method, with the output of each case showing a reasonable level of accuracy in the phase processed. It can be concluded that the whole proposed image segmentation approach is sensible and that each phase of this approach is appropriate to assist the proposed approach to detect the outlines of the river in the target image. The only limitation of this approach is we could not generalize the set of parameters that can be used for all image segmentation and background removal since the images where collected from various resources and under different environmental conditions. The lack of accessibility of a database of images collected from a unified sensor in a limitation that needs to be investigated. 
Carey Ciaburri et al.

(a) Original

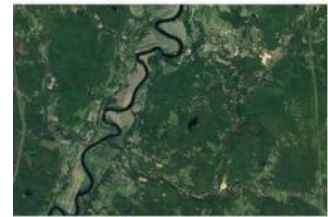

(b) Decorrelation

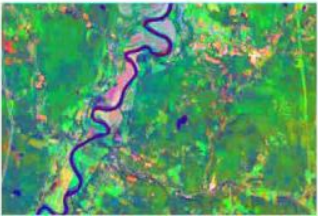

(c) Segmented

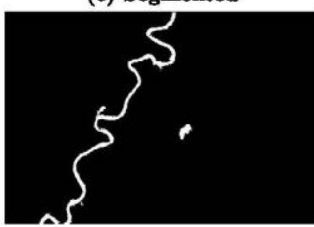

(d) Outlined

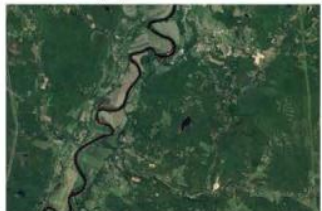

Figure 4 Image segmentation for satellite image 1: (a) original image (b) decorrelated image (c) segmented image (d) outlines of the original image

(a) Original

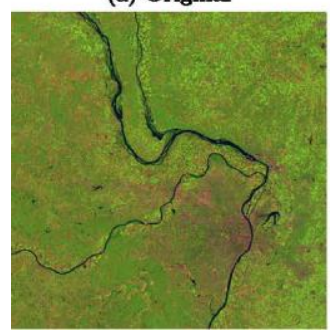

(b) Decorrelation

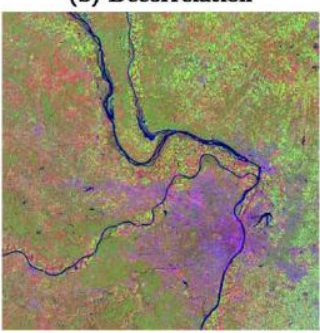

(c) Segmented

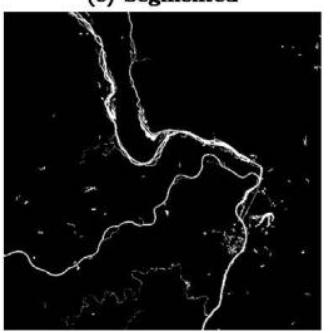

(d) Outlined

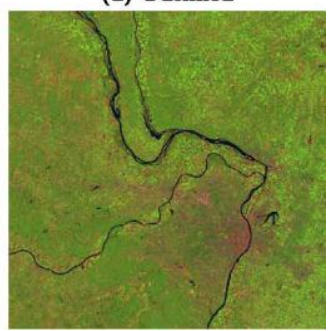

Figure 5 Image segmentation for satellite image 2: (a) original image (b) decorrelated image (c) segmented image (d) outlines of the original image

(a) Original

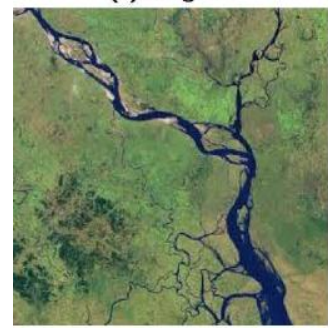

(b) Decorrelation

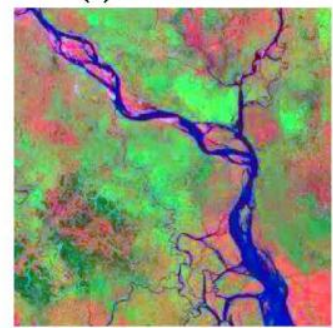

(c) Segmented

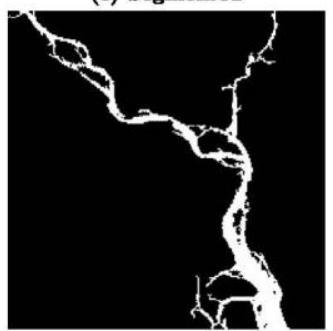

(d) Outlined

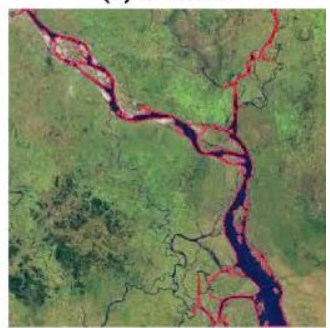

Figure 6 Image segmentation for satellite image 3: (a) original image (b) decorrelated image (c) segmented image (d) outlines of the original image

(a) Original

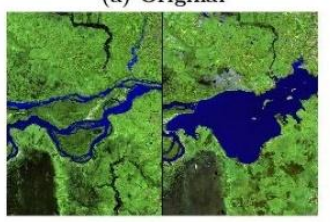

(b) Decorrelation

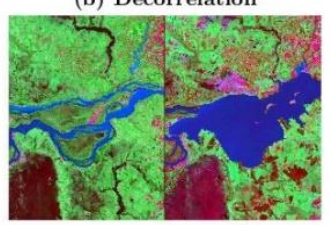

(c) Segmented

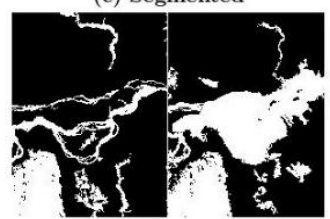

(d) Outlined

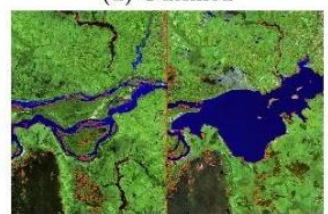

Figure 7 Image segmentation for satellite image 4: (a) original image (b) decorrelated image (c) segmented image (d) outlines of the original image

(a) Original

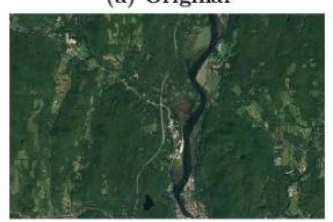

(b) Decorrelation

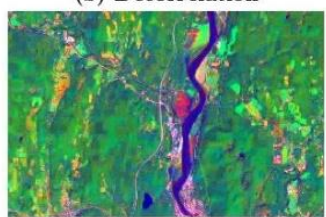

(c) Segmented

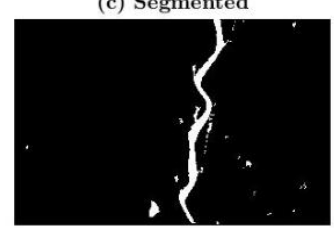

(d) Outlined

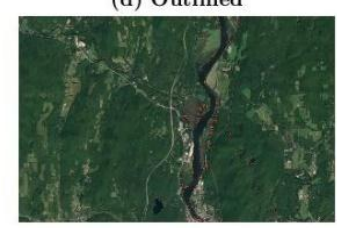

Figure 8 (a) Original satellite image (b) decorrelated image (c) segmented image (d) outlines of the original image 


\section{Conclusion and future work}

In this paper, we presented our initial idea using various color models based on image processing methodology to detect rivers from satellite images. The methodology was tested on a set of images collected for the Connecticut River in the state of Connecticut taken at a height of 14,000-15,000 meters. The decorrelation process adopted to enhance the color in the images helped to enhance the quality of the images and make it easier to segment the images. A different level of segmentation was adopted for better detection of rivers. This research could serve as a groundwork for future research on developing water resource management, which is crucial for future generations. We suggest that this methodology could be enhanced by using metaheuristic search techniques to better tune the segmentation filters to segment the rivers. Also, in further work, we will focus on the following trends: a more powerful segmentation approach to obtaining more accurate segments of the rivers, an enhancement model that is sufficiently adequate for the images, and a mechanism for extracting artificial and non-artificial objects of the rivers. These future directions can enhance the performance level of the proposed image segmentation method and promote work adequacy.

\section{Acknowledgment}

None.

\section{Conflicts of interest}

The authors have no conflicts of interest to declare.

\section{References}

[1] Stagl J, Mayr E, Koch H, Hattermann FF, Huang S. Effects of climate change on the hydrological cycle in central and eastern Europe. In managing protected areas in central and eastern Europe under climate change 2014 (pp. 31-43). Springer, Dordrecht.

[2] Rizvi IA, Mohan BK, Bhatia PR. Automatic object extraction using object based image classification technique from high resolution remotely sensed images. In proceedings of the international conference and workshop on emerging trends in technology 2010 (pp. 623-8)

[3] Hakdaoui $S$, Emran A. Extraction of water information based on sar radar and optical image processing: case of flood disaster in southern Morocco. In geospatial technology 2020 (pp. 15-29). Springer, Cham.

[4] Zhang F, Li J, Zhang B, Shen Q, Ye H, Wang S, Lu Z. A simple automated dynamic threshold extraction method for the classification of large water bodies from landsat-8 OLI water index images. International Journal of Remote Sensing. 2018; 39(11):3429-51.
[5] Zhang W, Hu B, Brown GS. Automatic surface water mapping using polarimetric SAR data for long-term change detection. Water. 2020; 12(3).

[6] $\mathrm{Hu} \mathrm{S}$, Qin J, Ren J, Zhao H, Ren J, Hong $\mathrm{H}$. Automatic extraction of water inundation areas using sentinel-1 data for large plain areas. Remote Sensing. 2020; 12(2).

[7] Al-Amri SS, Kalyankar NV. Image segmentation by using threshold techniques. arXiv preprint arXiv:1005.4020. 2010.

[8] Gong Z, Wang Q, Guan H, Zhou D, Zhang L, Jing R, et al. Extracting tidal creek features in a heterogeneous background using Sentinel-2 imagery: a case study in the Yellow River Delta, China. International Journal of Remote Sensing. 2020; 41(10):3653-76.

[9] Sakurai-Amano T, Onuki S, Takagi M. Automatic extraction of rivers in tropical rain forests from JERS1 SAR images using spectral and spatial information. In international geoscience and remote sensing symposium 2002 (pp. 3429-31). IEEE.

[10] Le TA, Lam DT, Vo P, Yoshitaka A, Le HB. Recover water bodies in multi-spectral satellite images with deep neural nets. In Proceedings of the ninth international symposium on information and communication technology 2018 (pp. 281-8).

[11] Bello MM, Nasidi NM, Shanono NJ. Remote sensing as a tool for irrigation water management.2014.

[12] Shah V, Choudhary A, Tewari K. River extraction from satellite image. International Journal of Computer Science Issues. 2011; 8(4):386-91.

[13] Zhaohui Z, Prinet V, Songde MA. Water body extraction from multi-source satellite images. In IGARSS 2003. International geoscience and remote sensing symposium. proceedings (IEEE Cat. No. 03CH37477) 2003 (pp. 3970-2). IEEE.

[14] Wang X, Xie H. A review on applications of remote sensing and geographic information systems (GIS) in water resources and flood risk management.

[15] Tymków P, Jóźków G, Walicka A, Karpina M, Borkowski A. Identification of water body extent based on remote sensing data collected with unmanned aerial vehicle. Water. 2019; 11(2).

[16] Randazzo G, Barreca G, Cascio M, Crupi A, Fontana M, Gregorio F, et al. Analysis of very high spatial resolution images for automatic shoreline extraction and satellite-derived bathymetry mapping. Geosciences. 2020; 10(5):172.

[17] Dhanachandra N, Manglem K, Chanu YJ. Image segmentation using K-means clustering algorithm and subtractive clustering algorithm. Procedia Computer Science. 2015; 54:764-71.

[18] Syrris V, Ferri S, Ehrlich D, Pesaresi M. Image enhancement and feature extraction based on lowresolution satellite data. Journal of Selected Topics in Applied Earth Observations and Remote Sensing. 2015; 8(5):1986-95.

[19] Jiang W, He G, Long T, Ni Y, Liu H, Peng Y, Lv K, Wang G. Multilayer perceptron neural network for surface water extraction in Landsat 8 OLI satellite images. Remote Sensing. 2018; 10(5). 
[20] Rishikeshan CA, Ramesh H. An ANN supported mathematical morphology based algorithm for lakes extraction from satellite images. ISH Journal of Hydraulic Engineering. 2018; 24(2):222-9.

[21] Miao Z, Fu K, Sun H, Sun X, Yan M. Automatic water-body segmentation from high-resolution satellite images via deep networks. IEEE Geoscience and Remote Sensing Letters. 2018; 15(4):602-6.

[22] Li K, Hu X, Jiang H, Shu Z, Zhang M. Attentionguided multi-scale segmentation neural network for interactive extraction of region objects from highresolution satellite imagery. Remote Sensing. 2020; 12(5):789.

[23] Meng L, Zhang Z, Zhang W, Ye J, Wu C, Chen D, et al. An automatic extraction method for lakes and reservoirs using satellite images. IEEE Access. 2019; 7:62443-56.

[24] Feng W, Sui H, Huang W, Xu C, An K. Water body extraction from very high-resolution remote sensing imagery using deep U-Net and a superpixel-based conditional random field model. IEEE Geoscience and Remote Sensing Letters. 2018; 16(4):618-22.

[25] Rishikeshan CA, Ramesh H. An automated mathematical morphology driven algorithm for water body extraction from remotely sensed images. ISPRS Journal of Photogrammetry and Remote Sensing. 2018; 146:11-21.

[26] Patel HJ, Dabhi VK, Prajapati HB. River water pollution analysis using high resolution satellite images: a survey. In 5th international conference on advanced computing \& communication systems (ICACCS) 2019 (pp. 520-5). IEEE.

[27] Navarro JC, Salazar-Garibay A, Téllez-Quiñones A, Orozco-del-Castillo M, López-Caloca AA. Inland water body extraction in complex reliefs from Sentinel-1 satellite data. Journal of Applied Remote Sensing. 2019; 13(1).

[28] Dereli MA, Tercan E. Assessment of shoreline changes using historical satellite images and geospatial analysis along the lake salda in turkey. Earth Science Informatics. 2020:1-10.

[29] Wang Y, Li Z, Zeng C, Xia GS, Shen H. An urban water extraction method combining deep learning and google earth engine. Journal of selected topics in applied earth observations and remote sensing. 2020; 13:768-81.

[30] Liu Y, Zhang P, He Y, Peng Z. River detection based on feature fusion from synthetic aperture radar images. Journal of Applied Remote Sensing. 2020; 14(1):016505.

[31] Nguyen UN, Pham LT, Dang TD. An automatic water detection approach using Landsat 8 OLI and google earth engine cloud computing to map lakes and reservoirs in New Zealand. Environmental monitoring and assessment. 2019; 191(4):235.

[32] Abubakar FM. Study of image segmentation using thresholding technique on a noisy image. International Journal of Science and Research. 2013:49-51.

[33] Sekertekin A. A survey on global thresholding methods for mapping open water body using sentinel-
2 satellite imagery and normalized difference water index. Archives of Computational Methods in Engineering. 2020:1-3.

[34] Japitana MV, Demetillo AT, Burce ME, Taboada EB. Catchment characterization to support water monitoring and management decisions using remote sensing. Sustainable Environment Research. 2019; 29(1):1-10.

[35] Guo Q, Wu X, Sang X, Fu Y, Zang Y, Gong X. An integrated study on change detection and environment evaluation of surface water. Applied Water Science. 2020.

[36] Karvelis PS, Fotiadis DI. A region based decorrelation stretching method: Application to multispectral chromosome image classification. In international conference on image processing 2008 (pp. 1456-9). IEEE.

[37] Zhao M, Zhang C, Zhang W, Li W, Zhang J. Decorrelation-stretch based cloud detection for total sky images. In visual communications and image processing (VCIP) 2015 (pp. 1-4). IEEE.

[38] Campbell NA. The decorrelation stretch transformation. International Journal of Remote Sensing. 1996; 17(10):1939-49.

[39] Comaniciu D, Meer P. Robust analysis of feature spaces: color image segmentation. In proceedings of computer society conference on computer vision and pattern recognition 1997 (pp. 750-5). IEEE.

[40] Sheta A, Alkasassbeh M, Braik M, Ayyash HA. Detection of oil spills in SAR images using threshold segmentation algorithms. International Journal of Computer Applications. 2012; 57(7):10-15.

[41] Sezgin M, Sankur B. Survey over image thresholding techniques and quantitative performance evaluation. Journal of Electronic imaging. 2004; 13(1):146-66.

[42] Vala HJ, Baxi A. A review on Otsu image segmentation algorithm. International Journal of Advanced Research in Computer Engineering \& Technology. 2013; 2(2):387-9.

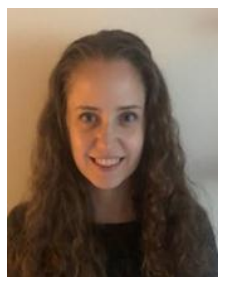

Carey Ciaburri is a Graduate Student working on a Master's degree in Applied Physics at Southern Connecticut State University. She received a B.S. degree in Earth Science with a concentration in Geology from Southern Connecticut State University in 2017.

Email: shetaa1@southernct.edu

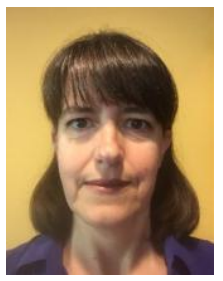

Monica Kiehnle-Benitez is working on her Master's in Applied Physics at Southern Connecticut State University. She graduated from the Instituto Tecnologico y de Estudios Superiores de Monterrey, Campus Morelos, Mexico, in 1993, with a degree in Industrial and Systems Engineering. 


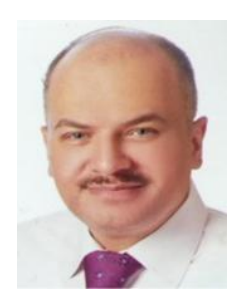

Alaa Sheta is a Professor of Computer Science at Southern CT State University. He received his B.E., M.Sc. degrees in Electronics and Communication Engineering from the Faculty of Engineering, Cairo University in 1988, and 1994, respectively. In 1997, he received his

Ph.D. from the Computer Science Department, School of Information Technology and Engineering, George Mason University, Fairfax, VA, USA. His research record includes more than 150 conference and journal publications, 6 book chapters, and 3 books. He is an Associate Editor of the International Journal of Advanced Computer Science and Applications (IJACSA) and the International Journal of Computational Complexity and Intelligent Algorithms.

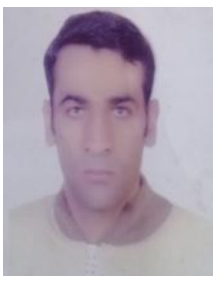

Malik Braik received a B.Sc. degree in Electrical engineering from the Faculty of Engineering, Jordan University of Science and Technology, Jordan in 2000. Five years later, he received his M.Sc. degree in Computer Science from the Department of Information Technology, Al-Balqa Applied University, Jordan. Nine years later, he received his Ph.D. in Computer Engineering from the University of Birmingham, the U.K. He has more than 40 publications. His research interests include Computer Vision, Pattern Recognition, Evolutionary Computation, Control, And System Identification, Computational Intelligence, and Image Processing. He is currently an Assistant Professor at the Department of Computer Science, Prince Abdullah bin Ghazi Faculty of Communications and Information Technology, Al-Balqa Applied University, Al-Salt, Jordan. 